\title{
HOMOZYGOUS FUKUTIN MISSENSE MUTATION IN TWO MEXICAN SIBLINGS WITH DILATED CARDIOMYOPATHY
}

\author{
María T. Villarreal-Molina ${ }^{1}$, Sandra Rosas-Madrigal ${ }^{1}$, Enrique López-Mora ${ }^{2}$, Ana L. Calderón- \\ Avila ${ }^{1}$, Hugo Rodríguez-Zanella ${ }^{2}$, Sandra Romero-Hidalgo ${ }^{1}$, Rigoberto Rosendo-Gutierrez ${ }^{1}$, \\ and Alessandra Carnevale ${ }^{1,2, *}$
}

${ }^{1}$ Instituto Nacional de Medicina Genómica, Mexico City; ${ }^{2}$ Instituto Nacional de Cardiología "Ignacio Chávez", Mexico City, Mexico

\begin{abstract}
Background: Fukuyama congenital muscular dystrophy (FCMD) is the most common form of a group of autosomal recessive disorders characterized by altered $\alpha$-dystroglycan glycosylation and caused by FKTN gene mutations. However, mutations of this gene may cause a broad range of phenotypes, including Walker-Warburg syndrome, muscle-brain-eye disease, FCMD, limbgirdle muscular dystrophy without mental retardation, and cardiomyopathy with no or minimal skeletal muscle weakness. Objective: Our purpose was to describe two siblings who died at a young age with dilated cardiomyopathy (DCM), no muscle weakness, or atrophy, and were homozygous for a FKTN missense mutation. Methods: Site-directed next-generation sequencing (NGS) was performed. Pathogenicity of variants of interest was established according to the American College of Medical Genetics (ACMG) criteria, and all available first-degree relatives were screened for mutations by Sanger sequencing. Results: NGS revealed a homozygous FKTN variant in the index case (p.Gly424Ser, rs752358445), classified as likely pathogenic by ACMG criteria. Both parents and an unaffected brother were heterozygous carriers. Since the siblings had no apparent skeletal muscle weakness or central nervous system involvement, FKTN mutations were not initially suspected. Conclusions: This is the first report demonstrating that heterozygous individuals for the FKTN p.Gly424Ser mutation were healthy, while two homozygous brothers suffered severe DCM, strongly suggesting that this FKTN mutation is a rare cause of autosomal recessive DCM. (REV INVEST CLIN. 2021;73(3):XX-XX)
\end{abstract}

Key words: Fukutin. Dilated cardiomyopathy. Fukuyama congenital muscular dystrophy. FKTN missense mutation.

\section{INTRODUCTION}

Fukuyama congenital muscular dystrophy (FCMD) is the most common form of a group of autosomal recessive disorders characterized by altered $\alpha$-dystroglycan glycosylation and caused by FKTN gene mutations.

*Corresponding author:

Alessandra Carnevale

E-mail: acarnevale@inmegen.gob.mx
However, FKTN gene mutations may cause a broad spectrum of clinical manifestations. The most severe phenotype is Walker-Warburg syndrome (WWS), characterized by type 2 lissencephaly, cerebellar malformation, hydrocephalus, retinal and anterior eye chamber malformations, and congenital muscular dystrophy.

0034-8376 / (c) 2020 Revista de Investigación Clínica. Published by Permanyer. This is an open access article under the CC BY-NC-ND license (http://creativecommons.org/licenses/by-nc-nd/4.0/). 
Most affected infants die during the $1^{\text {st }}$ year of life ${ }^{1}$. FCMD is also a severe phenotype with severe congenital muscle wasting, structural brain alterations, cognitive impairment, and epilepsy. The clinical course is helplessly progressive, and death occurs at an average age of 16 years ${ }^{2,3}$. Limb-girdle muscular dystrophy without cognitive impairment is milder ${ }^{4}$, and cardiomyopathy with no or minimal skeletal muscle weakness is the mildest phenotype ${ }^{5,6}$.

The FKTN gene locus is $9 \mathrm{q} 31$, it has 10 exons and encodes a glycosyltransferase active in the Golgi complex called fukutin, involved in dystroglycan glycosilation ${ }^{7}$. FKTN mRNA expression patterns in the human brain suggest the protein may influence neuronal migration ${ }^{8,9}$. Fukutin is expressed ubiquitously in human tissues, although many aspects of its function in various tissues remain unknown ${ }^{10,11}$.

FCMD is the second most frequent form of childhood muscular dystrophy in Japan. Most Japanese patients are homozygous for a founder mutation consisting of a $3 \mathrm{~kb}$ insertion into the 3 ' untranslated region of the FKTN gene ${ }^{12}$. Clinically, these patients have early disease onset, hypotonia, generalized muscle weakness, structural brain malformations, and cognitive impairment ${ }^{13}$. The first non-Japanese patient with a FKTN mutation was reported by Silan et al. ${ }^{14}$, a Turkish infant born to consanguineous parents with macrocephaly, bilateral buphthalmos, cataracts, hypotonia, and dyspnea, who died 10 days after birth. Molecular analysis revealed a homozygous $1 \mathrm{bp}$ insertion within exon five of the FKTN gene, causing a frameshift and a premature stop codon at amino acid position 157 . Shortly after, another Turkish infant with WalkerWarburg syndrome born to consanguineous parents was found to be homozygous for a nonsense FKTN gene mutation (c.345_346GC; p.Gln116Ter) ${ }^{15}$. He had hypotonia, bilateral corneal clouding, severe hydrocephalus, brain stem hypoplasia, and absent corpus callosum. An increasing number of FKTN mutations have recently been reported in non-Japanese patients, with phenotypes ranging from WWS, muscle-brain-eye disease, FCMD, and at the mild end of the spectrum, limb-girdle muscular dystrophy with no mental retardation and cardiomyopathy with no or minimal skeletal muscle weakness ${ }^{16,17}$.

Our purpose was to describe two Mexican siblings with dilated cardiomyopathy (DCM) and no signs of skeletal muscle weakness or atrophy, found to be homozygous for a very low-frequency FKTN missense variant.

\section{MATERIALS AND METHODS}

\section{Patients}

We studied two Mexican male siblings who died from heart failure and DCM at ages 20 and 21 years. Their parents were consanguineous (first cousins) and had two other apparently healthy offspring (Fig. 1). The index case (IV-3) had a normal childhood with normal developmental milestones. He was first referred to the National Institute of Cardiology in Mexico City at age 17 years due to progressive exercise intolerance, dyspnea, and inferior limb edema. Physical examination showed jugular venous distention, hepatojugular reflex, mitral and tricuspid regurgitant murmurs, hepatomegaly, and ascites. Echocardiography revealed severe mitral and tricuspid regurgitation, as well as biventricular enlargement and dysfunction. Cardiac magnetic resonance (CMR) showed enlargement of right and left cavities (left ventricle end-diastolic and systolic volumes were $216 \mathrm{ml}$ and $182 \mathrm{ml}$, respectively), biventricular dysfunction, and myocardial fibrosis (Fig. 2). Left ventricle ejection fraction (LVEF) and right ventricle ejection fraction (RVEF) were reduced ( $16 \%$ and $15 \%$, respectively). Pharmacological treatment initially caused clinical improvement; however, 6 months later, the patient developed acute heart failure, renal failure, and hepatic congestion. Treatment with diuretics and intravenous inotropes led to temporary improvement, but episodes of decompensated heart failure continued. He was admitted 4 months later with cardiogenic shock and died at age 21 years.

The youngest sibling (IV-4) had a similar medical history, with an apparently healthy childhood and adolescence. At age 18 years, he developed progressive dyspnea. CMR showed enlargement of both right and left cavities, biventricular dysfunction, reduced LVEF and RVEF ( $13 \%$ and $27 \%$, respectively), and diffuse fibrosis of septal predominance (Fig. 2). While receiving pharmacological treatment for heart failure, he died of sudden cardiac death at age 20 years. He had no clinical signs of muscular dystrophy, and unfortunately, no muscle biopsies were available to study $\alpha$-dystroglycan glycosylation. 
Figure 1. Family pedigree. DNA samples were available only for individuals III-1, III-2, IV-2, IV-3, and IV-4.

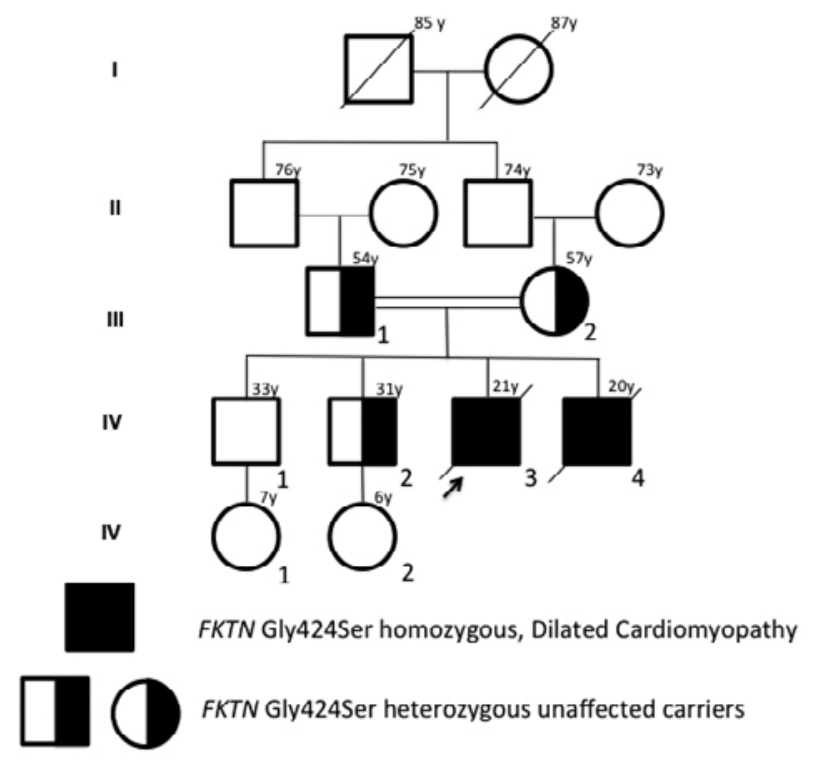

Figure 2. Cardiac magnetic resonance of the index case and younger sibling. Four chamber view (Panel A) and short-axis view (Panel B) of the index case showing enlargement of both right and left cavities. Late gadolinium intramyocardial enhancement was found in the four chamber (Panel E) and short-axis view (Panel F), compatible with non-ischemic myocardial fibrosis. Four chamber view (Panel C) and short-axis view (Panel D) of the younger sibling showing very similar changes, also with nonischemic myocardial fibrosis in the four chambers (Panel E) and short-axis view (Panel F). RA: right atrium; RV: right ventricle; LA: left atrium; LV: left ventricle.

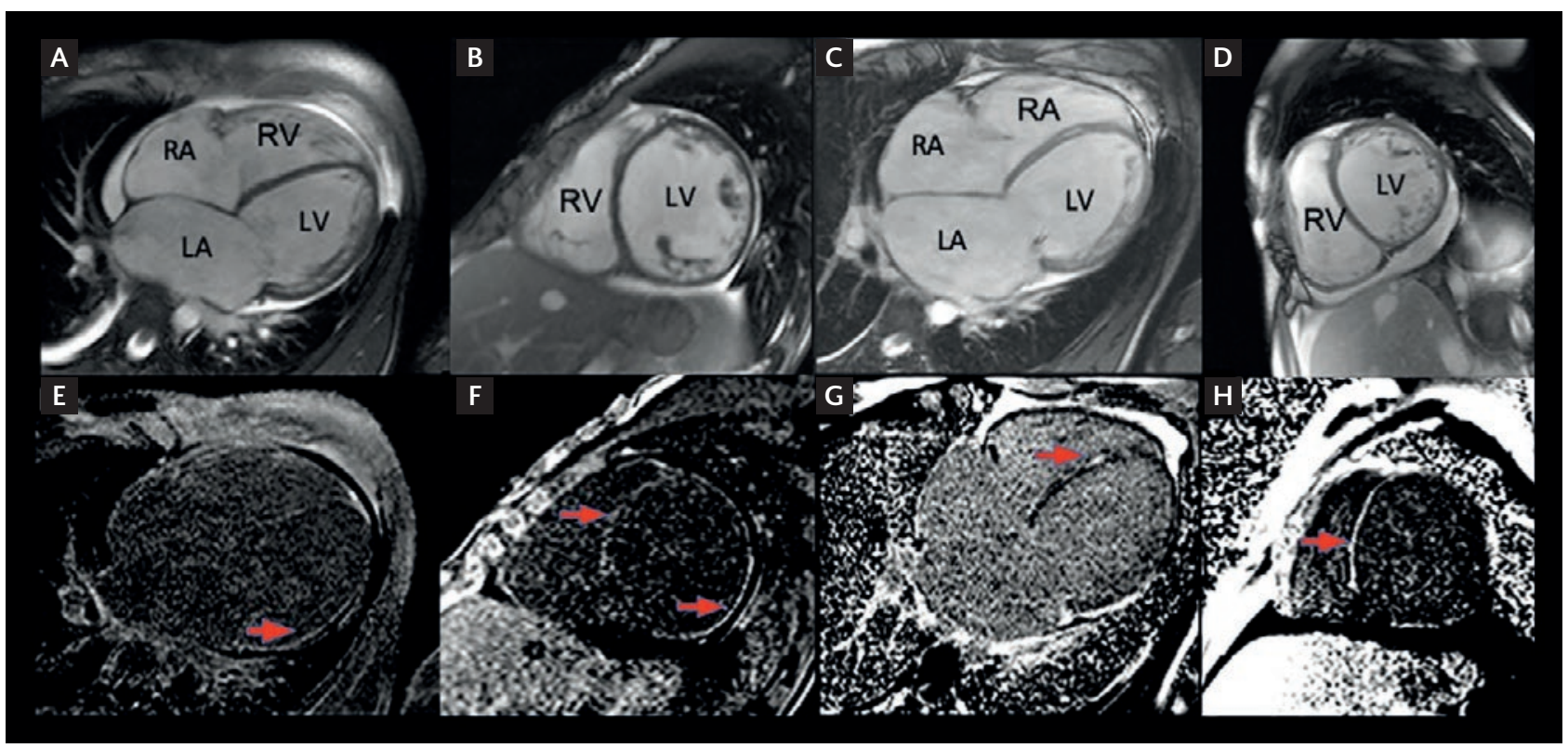

\section{Methods}

High-quality DNA samples previously extracted from blood of affected siblings, both parents and one unaffected sibling, were available for analysis. Targeted next-generation sequencing (NGS) was performed using TruSight-Cardio sequencing kit (Illumina) in a MiSeq System (Illumina). Post-run sequencing 
Figure 3. FKTN c.1270G>A (Gly424Ser) mutation. (A) Integrated Genome Viewer screenshot of the homozygous c.1270G>A mutation found in the index case. (B) Electropherogram confirming the homozygous C.1270G>A mutation, also found in the affected sibling (IV-4). (C) Electropherogram showing the heterozygous C.1270G>A mutation found in both parents and an unaffected sibling.

A

\section{B}

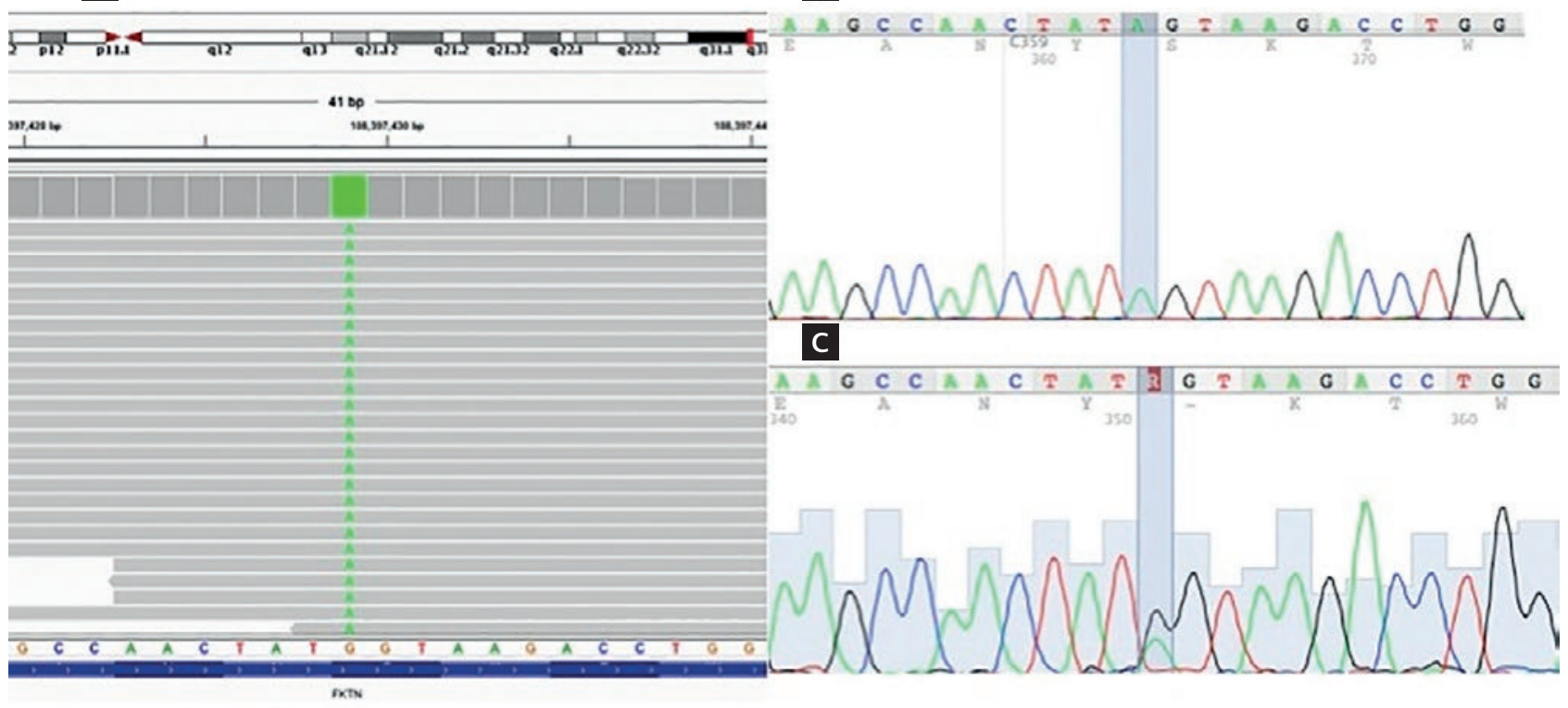

quality was assessed with FastQC (Babraham Bioinformatics, UK). Sequence reads were aligned with BWA Enrichment v2.1.0 and variant calling was performed using GATK v4.0. Variants were annotated with ANNOVAR (wannovar.wglab.org) and variant effect predictor (grch37.ensembl.org/Homo_sapiens/ Tools/VEP). In silico programs included in ANNOVAR annotation were used to predict functional consequences of the missense change and MaxEntScan (hollywood.mit.edu/burgelab/maxent/Xmaxentscan_scoreseq.html) was used to predict splice site alterations. Variants of interest found in DCMrelated genes were classified according to the American College of Medical Genetics criteria ${ }^{18}$ as benign, likely benign, of unknown clinical significance (VUS), likely pathogenic (LP) or pathogenic $(P)$, with the help of Varsome ${ }^{19}$. Variants of interest were confirmed by capillary sequencing and were screened in all firstdegree relatives.

The study was approved by the Comité de Ética en Investigación (Ethics Committee) of the Instituto Nacional de Medicina Genómica and the Instituto Nacional de Cardiología Ignacio Chávez, Mexico City, Mexico. All the family members participating in the study provided informed consent.

\section{RESULTS}

NGS of the index case (IV-3) revealed a homozygous mutation in the FKTN gene: NM_001198963.2: c.1270G>A p.Gly424Ser (rs752358445), causing a substitution of glycine (a non-polar aliphatic amino acid) for serine (a polar hydrophilic amino acid) at position 424 within the catalytic domain of the fukutin protein. Capillary sequencing confirmed the index case and his affected sibling was homozygous for this mutation, while both parents and an unaffected sibling (IV-2) were found to be asymptomatic FKTN Gly424Ser heterozygous carriers (Fig. 3). No other family members were available for molecular analysis.

In silico programs, SIFT, Polyphen2, LRT, Mutation Taster, Provean, M-CAP, and Fathmm-MKL predicted the functional consequence of the amino acid substitution as damaging, while only two in silico programs (MetSVM and FATHMM) predicted the change as tolerated. Interestingly, in addition to the amino acid substitution, a loss of function splice site alteration was predicted by MaxEntScan for 3 of the 9 FKTN transcripts (ENST00000357998.5, ENST00000448551.2, and ENST00000457847.1). 
The Gly424Ser substitution was classified as likely pathogenic, as the following AMCG criteria were met: moderate evidence of pathogenicity 2 (PM2: absent from controls or at extremely low frequency if recessive in Exome Sequencing Project, 1000 Genomes Project, or Exome Aggregation Consortium), and supporting evidence of pathogenicity $1,2,3$, and 4 (PP1: cosegregation with disease in multiple affected family members in a gene definitively known to cause the disease; PP2: missense variant in a gene that has a low rate of benign missense variation and in which missense variants are a common mechanism of disease; PP3: multiple lines of computational evidence support a deleterious effect on the gene or gene product; and PP4: patient's phenotype or family history is highly specific for a disease with a single genetic etiology). Considering the possibility of a splicesite alteration, the mutation would also be classified as likely pathogenic.

\section{DISCUSSION}

The clinical findings of the siblings described here differ from FCMD, which is characterized by severe muscle wasting, hypotonia, and cognitive impairment. Our patients suffered severe DCM, rapidly progressive heart failure, and died at young age. However, because they showed no apparent signs of skeletal muscle weakness or neurologic symptoms, FKTN mutations were not suspected and muscle biopsies were not performed. Notably, two unrelated DCM patients without neurological symptoms who developed muscle weakness during adulthood were previously described. They were compound heterozygous for the FKTN $3 \mathrm{~kb}$ insertion and one of two FKTN missense variants ( $p . Q 358 \mathrm{P}$ and $p . R 179 T)^{6}$.

In 2009, Arimura et al. ${ }^{17}$ further explored FKTN mutations in a group of 172 patients with DCM. The authors found a patient with mild muscular dystrophy and hyperCKemia but no brain involvement. This patient was compound heterozygous for FKTN mutations, the 3-kb insertion, and a missense Cys101Phe mutation. In addition, two DCM patients and three control subjects were heterozygous for the insertion and a normal FKTN allele, indicating that heterozygosity for the insertion itself is not associated with DCM. These observations suggest that compound heterozygous FKTN mutations could be a rare cause of DCM.

Nonsense and missense FKTN mutations may cause major damage to the fukutin protein and produce severe phenotypes. On the other hand, although the molecular mechanisms of FKTN mutations associated with DCM are unclear, fukutin function may be partially preserved in skeletal muscle of patients with minimal or late-onset muscle weakness. Further analyses are needed to clarify the function of fukutin in the glycosylation of $\alpha$-DG in different organs $6,2,10,11,17$.

The FKTN Gly424Ser variant was found in only one Latino individual in the GnomAD database (https:// gnomad.broadinstitute.org/) and is reported as of VUS in ClinVar (www.ncbi.nlm.nih.gov/clinvar/). However, because screening of first-degree relatives in this family revealed that both affected siblings were homozygous, and heterozygous carriers were asymptomatic (supporting evidence of pathogenicity), the variant met AMCG criteria to be classified as likely pathogenic. Moreover, while ClinVar mentions the WWS as a possible phenotype, it is known that homozygous loss of function FKTN mutations cause severe phenotypes, but compound heterozygous and missense mutations may cause milder phenotypes. Isolated DCM is considered as the mildest phenotype caused by FKTN mutations, and because our patients died at young age, it is unknown whether they may have developed skeletal muscle weakness at a more advanced age.

This is the first report demonstrating that heterozygous individuals for the c.1270G>A p.Gly424Ser mutation in the FKTN gene were healthy, while two homozygous brothers presented severe DCM, strongly suggesting that this mutation is a rare cause of autosomal recessive DCM.

\section{ACKNOWLEDGEMENTS}

The authors thank the financial support of the Consejo Nacional de Ciencia y Tecnología (CátedrasCONACYT) grant number 1703, and the Fondo Sectorial de Investigación en Salud y Seguridad Social (FOSISS) grant number 261670. 


\section{REFERENCES}

1. Godfrey C, Clement E, Mein R, Brockington M, Smith J, Talim B, et al. Refining genotype-phenotype correlations in muscular dystrophies with defective glycosylation of dystroglycan. Brain. 2007:130:2725-35.

2. Ismail S, Schaffer AE, Rosti RO, Gleesonb JG, Zaki MS. Novel mutation in the fukutin gene in an Egyptian family with Fukuyama congenital muscular dystrophy and microcephaly. Gene. 2014:539:279-82.

3. Kobayashi K, Kato R, Kondo-lida E, Taniguchi-Ikeda M, Osawa M, Saito K, Toda T. Deep-intronic variant of fukutin is the most prevalent point mutation of Fukuyama congenital muscular dystrophy in Japan. J Hum Genet. 2017;62:945-8.

4. Riisager M, Duno M, Hansen FJ, Krag TO, Vissing CR, Vissing J. A new mutation in the fukutin gene causing a late-onset limb girdle muscular dystrophy. Neuromuscul Disord. 2013;23:562-67.

5. Muller T, Krasniansky M, Witthaut R, Deschauer M, Zierz S. Dilated cardiomyopathy may be early sign of the C826A fukutin-related protein mutation. Neuromuscul Disord. 2005; 15:372-6

6. Murakami T, Hayashi YK, Noguchi S, Ogawa M, Nonaka I, Tanabe $Y$, et al. Fukutin gene mutations cause dilated cardiomyopathy with minimal muscle weakness. Ann Neurol. 2006;60:597-602.

7. Matsumoto $H$, Noguchi $S$, Sugie $K$, Ogawa $M$, Murayama $K$, Hayashi YK, et al. Subcellular localization of fukutin and fukutinrelated protein in muscle cells. J Biochem. 2004;135:709-12.

8. Haro C, Uribe ML, Quereda C, Cruces J, Martín-Nieto J. Expression in retinal neurons of fukutin and FKRP, the protein products of two dystroglycanopathy-causative genes. Mol Vision. 2018; 24:43-58.

9. Sasaki J, Ishikawa K, Kobayashi K, Kondo-li da E, Fukayama M, Mizusawa K, et al. Neuronal expression of the fukutin gene. Hum Mol Genet. 2000;9:3083-90.

10. Yamamoto T, Shibata N, Saito Y, Osawa M, Kobayashi M. Functions of fukutin, a gene responsible for fukuyama type congenital muscular dystrophy, in neuromuscular system and other somatic organs. Cent Nerv Syst Agents Med Chem. 2010 10:169-79.

11. Hiroi A, Yamamoto T, Shibata N, Osawa M, Kobayashi M. Roles of fukutin, the gene responsible for fukuyama-type congenital muscular dystrophy, in neurons: possible involvement in synaptic function and neuronal migration. Acta Histochem Cytochem. 2011:44:91-101.

12. Hayashi YK, Ogawa M, Tagawa K, Noguchi S, Ishihara T, Nonaka I, et al. Selective deficiency of alpha-dystroglycan in Fukuyama-type congenital muscular dystrophy. Neurology. 2001; 57:115-21.

13. Yis $U$, Uyanik G, Heck PB, Smitka $M$, Nobel $H$, Ebinger $F$, et al. Fukutin mutations in non-Japanese patients with congenital muscular dystrophy: less severe mutations predominate in patients with a non-Walker-Warburg phenotype. Neuromuscul Disord. 2011;21:20-30.

14. Silan F, Yoshioka M, Kobayashi K, Simsek E, Tunc M, Alper M, et al. A new mutation of the fukutin gene in a non-Japanese patient. Ann Neurol. 2003;53:392-6.

15. De Bernabé DB, van Bokhoven $H$, van Beusekom E, Van den Akker W, Kant S, Dobyns WB, et al. A homozygous nonsense mutation in the fukutin gene causes a Walker-Warburg syndrome phenotype. J Med Genet. 2003;40:845-8.

16. Costa C, Oliveira J, Gonçalves A, Santos R, Bronze-da-Rocha E, Rebelo $\mathrm{O}$, et al. A Portuguese case of Fukuyama congenital muscular dystrophy caused by a multi-exonic duplication in the fukutin gene. Neuromuscul Disord. 2013;23:557-61.

17. Arimura T, Hayashi YK, Murakami T, Oya Y, Funabe S, ArikawaHirasawa $\mathrm{E}$, et al. Mutational analysis of fukutin gene in dilated cardiomyopathy and hypertrophic cardiomyopathy. Circ J. 2009; 73:158-61.

18. Richards S, Aziz N, Bale S, Bick D, Das S, Gastier-Foster J, et al. Standards and guidelines for the interpretation of sequence variants: a joint consensus recommendation of the American college of medical genetics and genomics and the association for molecular pathology. Genet Med. 2015;17:405-24.

19. Kopanos C, Tsiolkas V, Kouris A, Chapple CE, Albarca-Aguilera $\mathrm{M}$, Meyer R, et al. VarSome: the human genomic variant search engine. Bioinformatics. 2018;35:1978-80. 